\title{
Vascular radiology in liver disease
}

\author{
LOUIS KREEL \\ M.D., M.R.C.P., F.F.R. \\ Royal Free Hospital, London, W.C.1
}

\begin{abstract}
Summary
Vascular radiology of the liver has increased in scope and function in recent years due mainly to the application of new techniques.

It is now possible to examine not only the inferior vena cava and the portal venous system, but also the hepatic veins and the coeliac axis and superior mesenteric artery. Hepatic vein occlusion, portal vein patency and collateral veins, as well as spaceoccupying lesions, can now be diagnosed with a fair degree of accuracy.

These techniques have also helped in the understanding of the altered haemodynamics of portal hypertension and can be used for treatment by intraarterial perfusion of chemotherapeutic substances.
\end{abstract}

\section{Introduction}

The full application of vascular radiology to the diagnosis of disease of the liver has come somewhat later than in other organs such as the kidneys and brain for two reasons: (1) the rapid development of liver biopsy and histological techniques appeared to make other anatomical methods of diagnosis superfluous and when any doubt existed laparotomy was undertaken; and (2) it required the introduction of selective arterial catheterization to show that sufficient detail could be obtained to render this a useful diagnostic tool. At present most liver units make extensive use, not only of spleno-portography but also of arteriography, hepatic venography and cavography. These methods of vascular radiology may not only provide an anatomical and pathological diagnosis but in many cases also demonstrate the complex altered haemodynamics which result from liver disease.

The particular examination or sequence of radiological examinations to be undertaken will depend not only on the clinical problem, but also on the availability of ancillary investigations such as isotope or ultrasonic scanning, the whims and fancies of particular clinicians and the desire of radiological departments to become involved in this work. As far as radiologists are concerned it is no longer a question of extraordinary skill or complex equip- ment because in most large hospitals there are 'arteriographers' available and the equipment has long since been installed.

The technical factors that have been responsible for the development of these procedures include the Seldinger method of percutaneous catheter insertion, the introduction of pre-formed radio-opaque catheters, non-toxic contrast medium, and the availability of image intensification and television monitoring. There is now little doubt that these techniques can be quickly mastered in a modern radiology department -the great mystique of selective arteriography has given way to a matter-of-fact approach with a consequent greatly increased demand in cases with liver disease.

The apparent complexity of vascular radiology of the liver stems from the possible anatomical approaches that can be used. Not only is it possible to visualize the hepatic veins, the splenic and portal venous system and the hepatic arteries but this can be achieved by different methods. Indications and contra-indications for these are fairly well defined and will be considered in each case. However, the actual techniques will be referred to only briefly as these are now fairly standard, considered in detail in most texts and in any case, like most practical procedures, are learnt by experience.

\section{Hepatic venography}

The three hepatic veins drain into the inferior vena cava, close to its entry into the right atrium and are best approached from the right medial basilic vein at the elbow via the subclavian vein, superior vena cava and right atrium. An end hole N.I.H. 7 or 8 cardiac catheter is preferred. The usual purpose of this is to obtain a wedged hepatic vein pressure to differentiate pre- from post-sinusoidal portal hypertension. By injecting contrast medium down the catheter (e.g. $20 \mathrm{ml}$ of $45 \%$ sodium diatrizoate) a wedged hepatic venogram can be obtained (Fig. 1). This examination has been used in cirrhosis where the portal vein often fills well in a retrograde fashion and in space-occupying lesions. However, it is most valuable in cases of hepatic vein occlusion (Budd- 


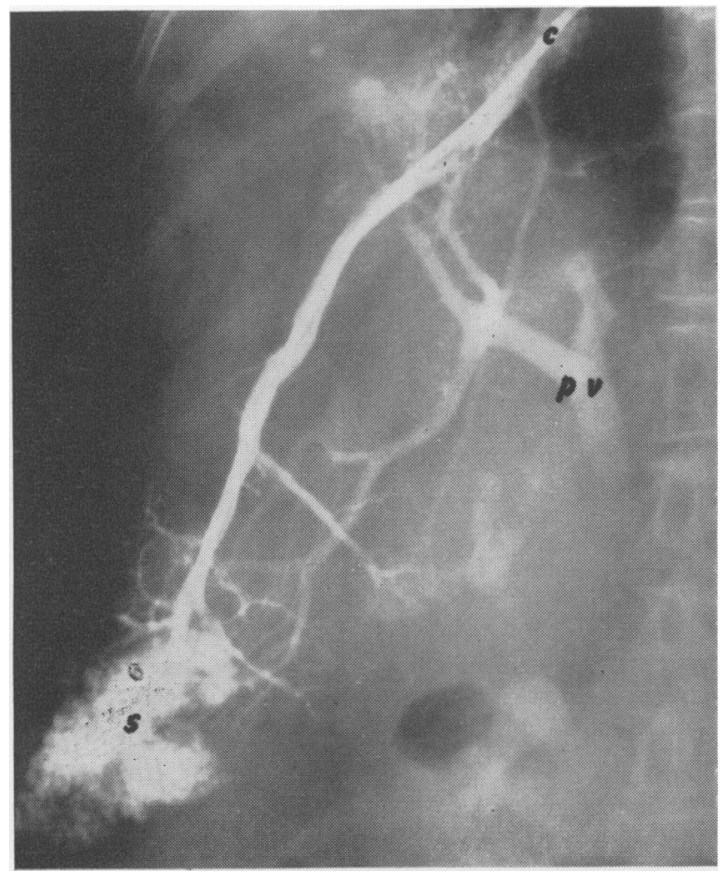

FIG. 1. Wedged hepatic venogram in cirrhosis of the liver. The sinusoidal pattern (s) is coarse and there is retrograde flow into the portal vein $(\mathrm{pv})$ to the hilum of the liver. Contrast medium is present around the catheter (c) lying in an hepatic vein.

Chiari syndrome) or veno-occlusive disease of the liver where specific diagnostic patterns may be produced, such as a short length stenosis of the hepatic vein with a leash of surrounding collateral vessels (Fig. 2a). The hepatic veins proximal to the block may be patent and dilated, filling out to the proximal tributaries. In other cases the larger hepatic veins may also be occluded. The appearance is then that of the demonstration of collateral hepatic veins forming a coarse network pattern without contrast medium being visible around the wedged catheter (Fig. 2b). The network pattern associated with the Budd-Chiari syndrome may also be comprised of very fine lines (Fig. 2c) and again no contrast will be seen in the vein around the catheter. Furthermore no contrast medium penetrates to the portal venous system. In veno-occlusive disease of the liver a similar network pattern may occur but the large hepatic veins remain patent and contrast medium can be seen around the catheter as it lies in an hepatic vein.

\section{Inferior vena-cavography}

The inferior vena cava is intimately related to the posterior aspect of the liver with liver tissue actually encircling this structure. It is thus not surprising that changes in the size and consistency of this organ are frequently reflected in the appearances produced by visualization of the inferior vena cava. In posteriorly lying space-occupying lesions the inferior vena cava is displaced, indicating the site of the lesion. On occasion this may be the only positive radiological examination. This occurs in medium sized lesions which impinge on the posterior surface of the liver, displacing the inferior vena cava forwards (Fig. 3). These posteriorly situated lesions also tend to cause obstruction of the vena cava with the production of a collateral circulation by way of the paraspinal and azygos systems. Large posterior space-occupying lesions produce most dramatic displacement of the vena cava which acts as an excellent marker in delineating their medial and posterior limits. In the Budd-Chiari syndrome, the generalized enlargement due to the swollen liver produces marked side-to-side compression of the inferior vena cava with very little change in its antero-posterior diameter. On the other hand, the small contracted cirrhotic liver produces circumferential narrowing of this vessel and its appearances are thus rather different in this condition. The compression caused by the Budd-Chiari syndrome, however, may become so severe as to lead to complete occlusion of the vena cava with a collateral network of channels being visualized.

The demonstration of the inferior vena cava is a simple procedure requiring only the percutaneous? catheterization of a femoral vein. Forty millilitres of $45 \%$ sodium diatrizoate is injected by hand and five films at 2 -sec intervals are exposed. This should be done in both frontal and lateral planes.

\section{Splenoportography}

Percutaneous splenovenography is the most frequently used vascular examination in liver disease and a well established procedure. The details of the technique are fully considered in standard texts. The basic precautions are to exclude a bleeding tendency and in no way to interfere with the free respiratory swing of the splenic needle. All manipulations associated with the needle must be done in an apnoeic phase.

The main indication for this examination is the pre-operative demonstration of the portal vein in cases of portal hypertension. For the successful performance of a portacaval shunt operation not only must the portal vein be of adequate calibre but the venous wall must be normal. The width of the portal vein in cases of portal hypertension is $2-3 \mathrm{~cm}$ with no or only slight tapering as it approaches the hilum of the liver. Any narrowing of the portal vein is usually associated with thickening of the wall due to periphlebitis. In portal hypertension there is an extensive collateral venous circulation with demonstration of the coronary vein (Fig. 4) and often also 


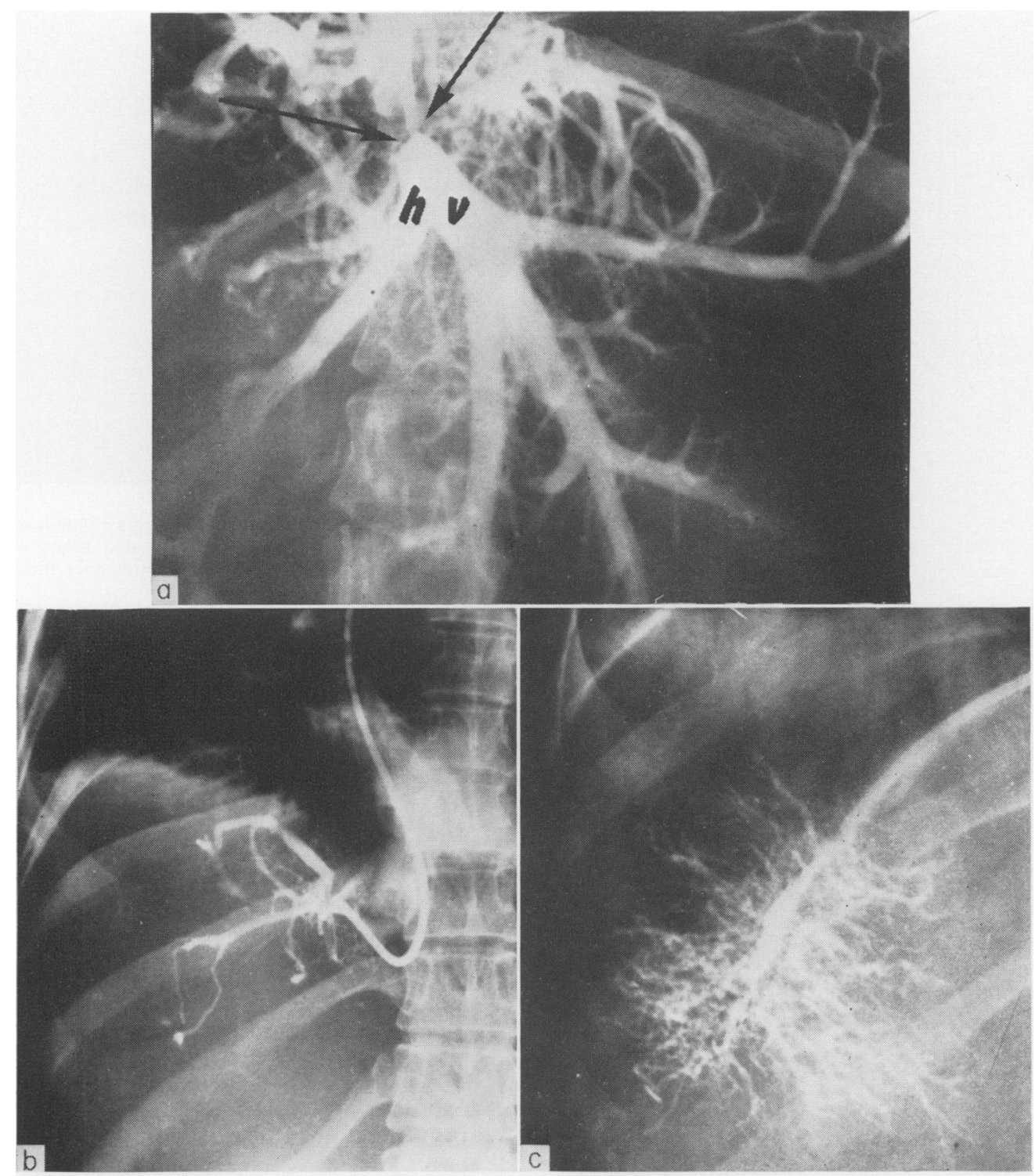

FIG. 2. Patterns seen in Budd-Chiari syndrome. (a) Hepatic venogram showing stenosis of an hepatic vein (hv) with dilatation of vein proximally and surrounding collateral veins. (b) Coarse network pattern of collateral veins. (c) Fine network pattern.

the umbilical and mesenteric veins. The collateral venous circulation in the abdomen can be so extensive as to produce a spontaneous 'porto-systemic' shunt with visualization of the inferior vena cava and this shunt can produce 'spontaneous' porto-systemic encephalopathy. The demonstration of the coronary collateral vein has recently become of greater significance, as this vein rather than the portal vein has been used for creating a venous shunt to the inferior vena cava for the relief of haematemesis.
Non-visualization of the portal vein may occur under two quite different circumstances. It may be due to block of the portal vein or merely to retrograde blood flow associated with portal hypertension. In both circumstances an extensive collateral venous circulation is present. Radiologically these conditions may be indistinguishable on the splenovenogram, however in occlusion of the portal and splenic vein there are 'bridging collaterals' which by-pass the site of obstruction to show the intrahepatic portal 

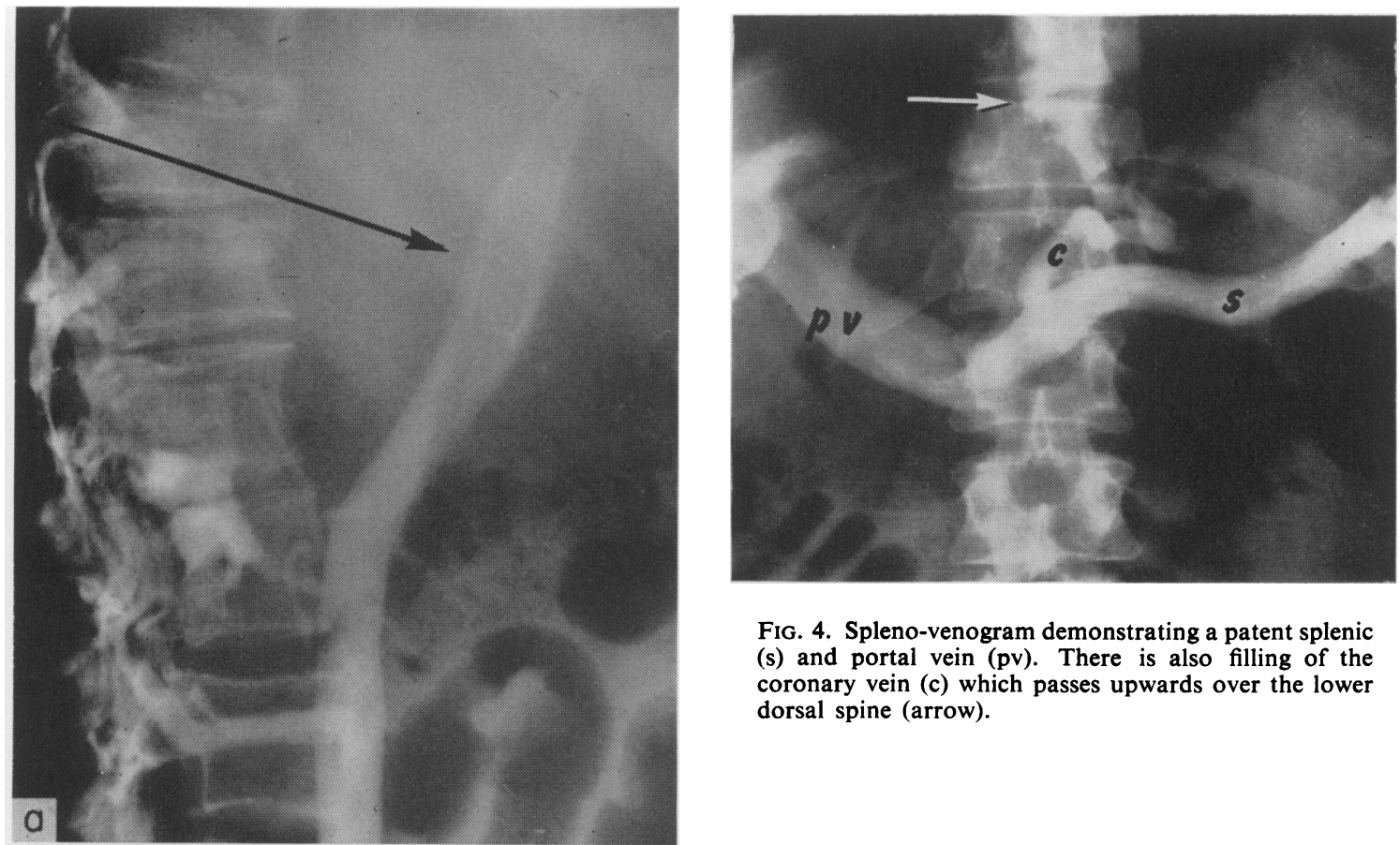

FIG. 4. Spleno-venogram demonstrating a patent splenic (s) and portal vein (pv). There is also filling of the coronary vein (c) which passes upwards over the lower dorsal spine (arrow).
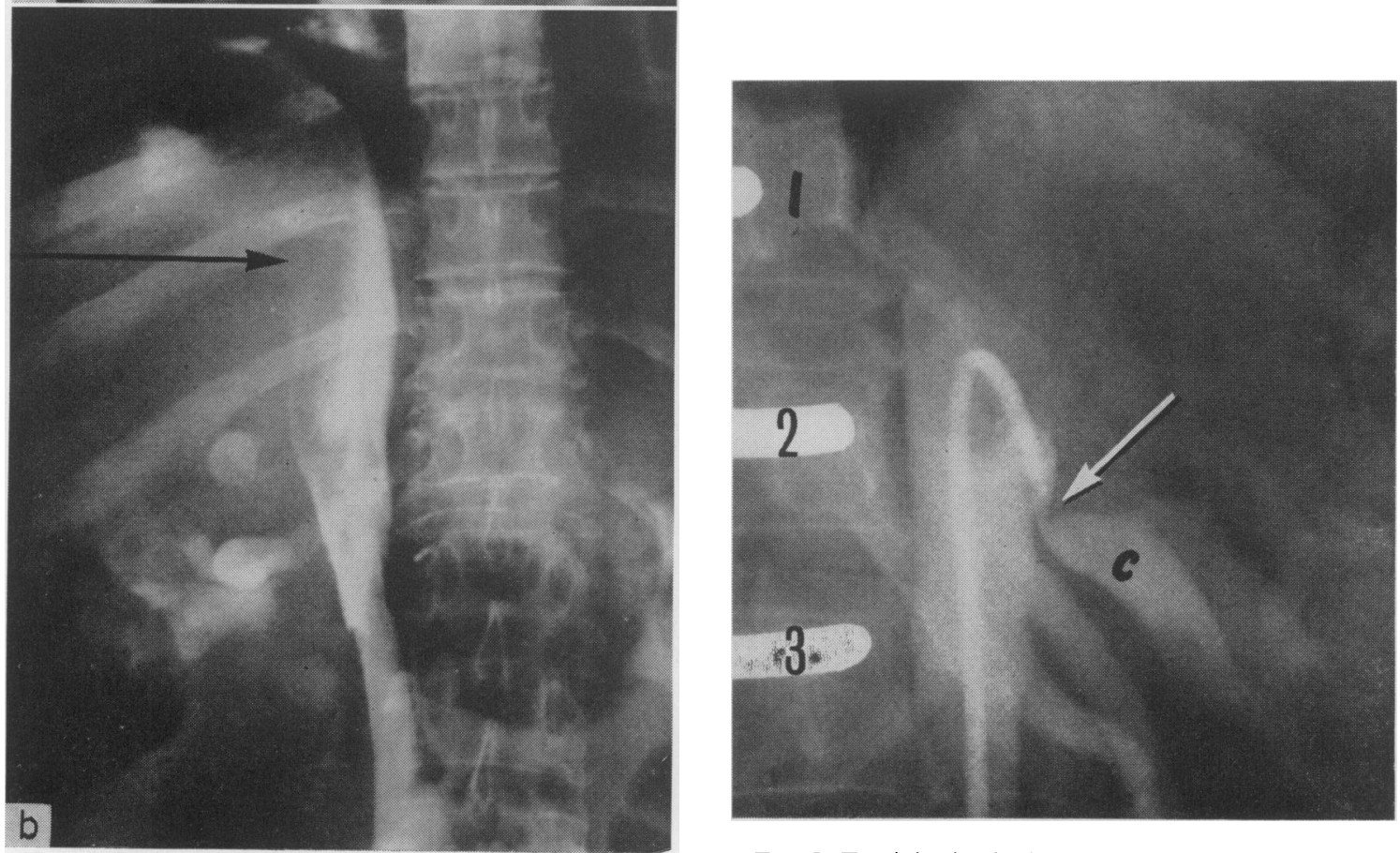

Fig. 3. Inferior vena cavogram showing forward displacement (a) and compression of the right lateral margin (b) of the inferior vena cava indicating a posteriorly lying liver mass which was due to actinomycosis. This lesion was not visible on splenoportography or arteriography.

FIG. 5. Test injection in the steep supine oblique position with the right shoulder raised. The lead skin markers are superimposed on the spine and it can be seen that the middle marker is near the level of the origin of the coeliac axis (c) which has a marked eccentric narrowing of the superior type due to the median arcuate ligament of the diaphragm. 
venous system. When this is of long standing it gives rise to numerous adjacent tortuous veins; the 'cavernous transformation' of the portal vein. If there is any doubt as to whether non-visualization of the portal vein is due to a true obstruction or to reversal of the blood flow, other procedures must be employed, such as wedged hepatic venography, mesenteric arterio-venography or umbilical portography.

Spleno-portography also produces other information although this is frequently of a less satisfactory nature because it is often either equivocal or better shown by other procedures. The demonstration of space-occupying lesions is such an example. These may be shown by isotope or ultrasonic scanning as well and often more clearly. Furthermore the nature of the lesion, whether solid or cystic, benign or malignant, cannot usually be distinguished on spleno-portography. It must be mentioned that hepatomas not infrequently cause intrahepatic thrombosis of the portal vein or filling defects within it. Thus a bare area in the liver, associated with non-filling of an intrahepatic branch of the portal vein or a filling defect within it, should indicate the strong possibility of a hepatoma. Large tumours or cysts in the left lobe of the liver cause downward displacement and may also produce narrowing of the splenic vein, thus indicating the site of such lesions.

The altered pattern of intrahepatic branching in cirrhosis producing a deficient branching pattern or 'tree in winter' appearance is seldom of prime importance as the diagnosis is essentially made from histological examination. However, the exact size of the liver can clearly be seen on spleno-portography and as this is difficult to estimate by other methods, can be of considerable importance.

\section{Umbilical vein portography}

The portal vein can be shown by passing a catheter along the umbilical vein remnant into the portal vein. The difficulty with this technique is that not infrequently the peritoneum is incised and, therefore, this procedure must be done in an operating theatre. It is, therefore, also unsuitable in the presence of ascites. However, the portal vein and its intrahepatic branches are densely shown by this method and it is particularly suitable for the study of the intrahepatic portal venous pattern. Following this procedure the umbilical vein becomes completely occluded and can thus only be used once on any patient. Portal vein sepsis has also been reported as a complication. It is particularly useful for space-occupying lesions and in showing the portal vein if this has not been demonstrated by splenovenography.

\section{Hepatic arteriography}

As with other organs, arteriography in liver disease may be used for the demonstration of vascular lesions as such, lesions within the liver itself especially space-occupying lesions, or for the demonstration of the portal venous system. However, in hepatic arteriography it is absolutely essential to perform selective arteriography and not just a free flush aortogram as with the latter method it is impossible to obtain adequate visualization of either the liver or the portal venous system. A free flush aortogram is occasionally required for the demonstration of the orifices of the coeliac axis and mesenteric arteries, but then this must be done as skyline views in either the steep oblique or lateral position.

Furthermore in hepatic arteriography much larger quantities of contrast medium are required than into other organs. The catheter must, therefore, be of adequate size for the quantity of contrast medium to be injected in a short enough period of time. The barium impregnated polyvinyl catheter (O.P.P. 205 or 240 -Portex) has been found suitable and $40 \mathrm{ml}$ of Conray ' 420 ' can be injected into the coeliac axis by hand with sufficient rapidity to obtain a good demonstration of the liver and portal vein.

\section{Arteriographic technique}

The details of the technique have been considered in detail in previous publications and will, therefore, only be briefly mentioned. Basically the technique is similar to selective catheterization of other organs performed by the Seldinger method, using a pre-formed opaque catheter. Once the catheter is inserted into the aorta the patient is turned into the supine oblique position with the right shoulder raised. Correctly placed opaque skin markers are extremely helpful in locating the orifices of the coeliac axis and superior mesenteric artery. If, however, there is any difficulty or delay in entering these vessels, a test injection must be done in the steep oblique position to show the position and calibre of these orifices and their relationship to the skin markers (Fig. 5). It then becomes a simple matter in most cases to direct the tip of the catheter into the appropriate artery.

The two common difficulties encountered are stenosis of the coeliac axis and anatomical variations. If a stenosis of the coeliac axis is encountered and this occurs in approximately $12 \%$ of cases, one can often still obtain an adequate examination by injecting the superior mesenteric artery. However, it is advisable to inject a slightly less concentrated contrast medium into this vessel (e.g. Hypaque $45 \%$ ) as peripheral pooling of the contrast medium may occur in the small bowel with poor visualization of the portal vein. To some extent this can be overcome by the use of $50 \mathrm{mg}$ of tolazoline diluted in $5 \mathrm{ml}$ in 
normal saline, being given in $2 \mathrm{sec}$ into the superior mesenteric artery about $30 \mathrm{sec}$ prior to the contrast medium. This is said to enhance visualization of the portal vein. In long standing coeliac axis stenosis, the gastroduodenal artery becomes greatly hypertrophied as it acts as the collateral channel to fill the hepatic and splenic arteries.

The other difficulty commonly encountered in selective catheterization of this region is due to the common occurrence $(20-25 \%)$ of anatomical variations. The commonest variation is that the right hepatic artery arises from the superior mesenteric but the whole hepatic artery may arise in this way. The splenic and left gastric arteries then have a separate origin from the aorta. Other variations include a completely separate origin of the splenic or the hepatic artery and a common origin for the coeliac axis and superior mesenteric artery. Unless these variations are borne in mind, much time may be wasted in searching for a non-existent orifice. Any doubts about the anatomical structure should be immediately resolved by recourse to a test injection and film.

Without going into any great detail, a few other technical factors of importance require mention. The film sequence used in any individual case is dependent on the nature of the clinical problem. To show lesions at the vascular orifices at the aorta the very early films (4/sec for $2 \mathrm{sec}$ ) are all important and exposures are begun as the first $5 \mathrm{ml}$ of contrast is injected by means of a pressure pump $\left(30 \mathrm{lb} / \mathrm{in}^{2}\right.$ in a $50-\mathrm{ml}$ syringe). For intrahepatic lesions early films are also required, but for somewhat longer ( $2 / \mathrm{sec}$ for $4 \mathrm{sec}$ ) and thus the first exposure is made as $20-25 \mathrm{ml}$ of contrast medium has been injected by hand. The later phase is also important as the portal vein should be demonstrated (one film every $2 \mathrm{sec}$ for twelve films). A steep supine oblique view with the left shoulder raised must also be done for intrahepatic lesions, but a shorter run is then adequate (2/sec for $4 \mathrm{sec}$ and $1 / \mathrm{sec}$ for $6 \mathrm{sec})$. In arterioportography the later phase is all important, but one film every other second is sufficient, however the examination should cover a period of $30 \mathrm{sec}$.

The injection of large quantities of contrast medium into the coeliac or mesenteric artery causes a burning pain which, however, lasts only 15-20 sec. The procedure can thus be done under local anaesthetic apart from the actual contrast medium injection which requires an ultra-short-acting anaesthetic such as Epontol.

\section{Uses and indications of hepatic arteriography}

The presence of a space-occupying lesion can be determined by isotope scanning if it is larger than 3 $\mathrm{cm}$ and similar sized lesions can be shown by ultrasonic scanning. The advantages of arteriography are that even smaller lesions can be shown provided that they are vascular and that in many cases arteriography will also indicate whether space-occupying lesions are benign or malignant. The other important indication is the demonstration of the portal or mesenteric vein as a pre-operative procedure in portal hypertension. Arterio-portography is preferred in cases where spleno-portography is contraindicated or cannot be done as with an abnormally prolonged prothrombin time, depressed platelet count or after splenectomy. Another indication for this procedure is when the portal vein has not been shown by spleno-portography. Superior mesenteric arterio-venography may then show either cavernous transformation of the portal vein, indicating portal vein obstruction, or a sufficiently large mesenteric vein to perform a shunt and even occasionally succeed in showing a portal vein not shown by splenoportography. Arterio-portography may also be used to show the patency of a portacaval shunt (Fig. 6), particularly in cases which have also had splenectomy.

Vascular lesions such as stenosis, aneurysms, angiomatous malformations and arterio-venous shunts are particularly well demonstrated. These lesions are often asymptomatic chance findings. In liver trauma the site of injury, active haemorrhage and a subor peri-capsular collection of blood may be shown. There has even been an example of the demonstration of active haemorrhage into the biliary system.

While the selective catheter is in position it is possible to undertake blood-flow studies by using radioactive xenon and surface counters. A further extension of the use of arteriography has been the catheter placement for the subsequent administra-

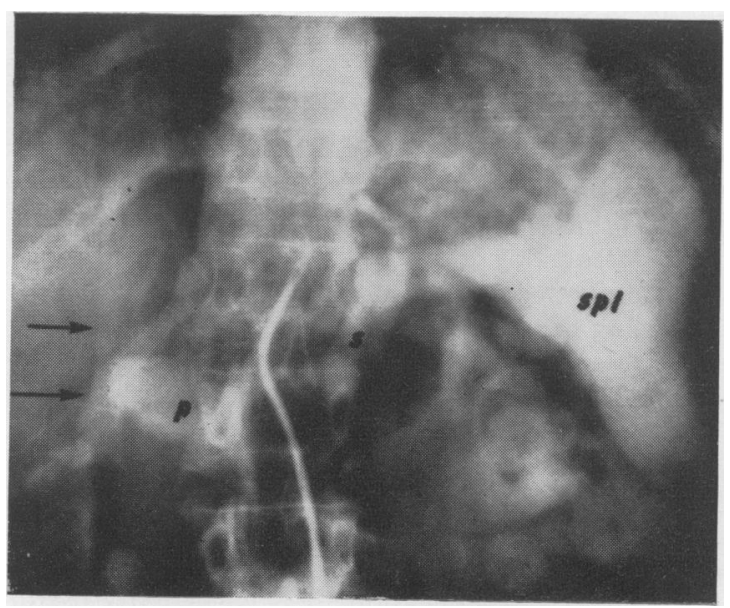

FIG. 6. Venous phase of arterio-venography showing the splenic (spl) and portal vein (p) draining into the inferior vena cava (arrows indicate right lateral margin). 
tion of intra-arterial infusion of cytotoxic agents. With care these catheters can be left in position for up to 3 weeks.

Arterio-venography has also been used to show the patency of a portacaval shunt and where malignant lesions have been demonstrated in other organs, such as the kidney, it is usually a simple matter to re-position the catheter to determine whether deposits are present in the liver. It may be mentioned that very definite changes may occur in the coeliac axis and its branches as a consequence of portal hypertension. Although at present these may only be considered as incidental observations, they do, however, further our understanding of the altered haemodynamics in portal hypertension.

\section{Space-occupying lesions}

\section{Benign conditions}

Once the presence of a mass lesion in the liver has been determined its nature may be ascertained by various tests including hydatid and amoebic complimentation fixation, the presence of foetoglobulins in hepatomas and by histology from needle biopsy. However, the exact extent of the lesion, its degree of vascularity and its relationship to the hepatic artery and portal vein can only be adequately shown by arteriography.

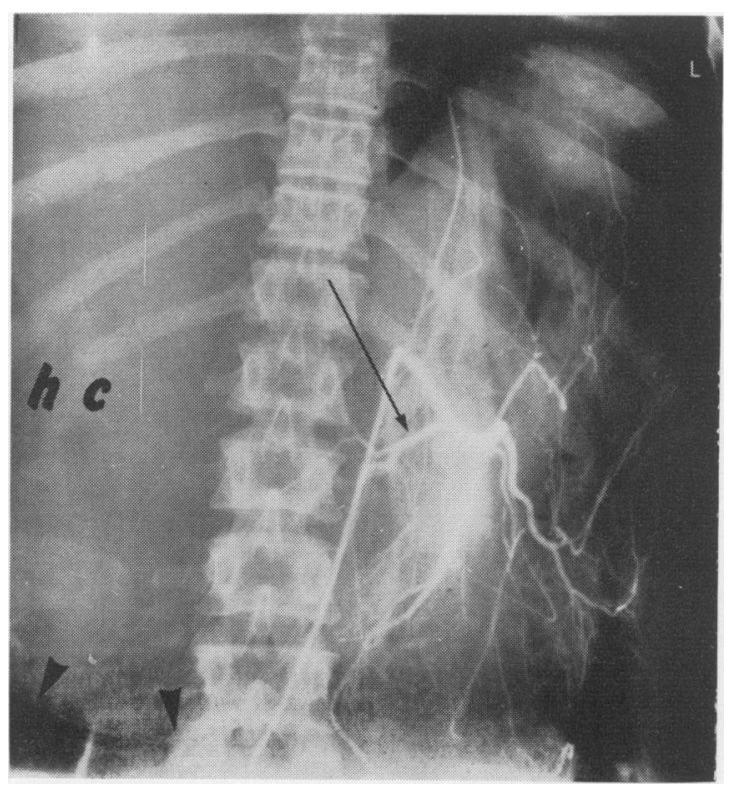

Fig. 7. Large hydatid cyst (hc) occupying the right lobe of the liver with compression and displacement of the left lobe. The lower margin is indicated by arrow heads. The right hepatic artery (arrow) and its branches are narrowed, displaced and stretched.
Hydatid disease of the liver may produce calcification particularly of a curvilinear type. On arteriography it produces a 'bare' area over which narrowed, curved and stretched vessels can be seen to be coursing (Fig. 7). The normal liver substance is displaced with compression of the arteries and veins which are equally affected. These cysts are usually best shown in the intermediate phase when both arteries and veins can be seen.

In the amoebic abscess the 'capsule' of the lesion is highly vascular and multiple small vessels are present, but these are regular in calibre and direction. In the one case of actinomycosis of the liver examined, the lesion was not visible arteriographically or on spleno-venography but was shown by forward displacement of the inferior vena cava.

Congenital cystic disease of the liver produces multiple small areas of vascular displacement which may be difficult to delineate. However, the associated cysts in the kidney may be obvious on pyelography and on arteriography.

The really difficult diagnostic problem is that of the benign adenoma or fibroma of the liver. These are extremely slow growing tumours which may reach a considerable size and are highly vascular on arteriography. These tumours may be the cause of hypertension. The extreme vascularity with large feeding arteries and irregular tortuous branches resembles the solitary hepatoma. The definitive diagnosis will, of course, be made on histology. The other benign tumour which can also be shown is the cavernous haemangioma of the liver. This lesion, however, produces characteristic angiographic appearances in that the slightly enlarged arteries are crowded together and there are varix-like spaces shown on the late phase. These contrast-filled spaces are clearly defined and retain the contrast medium for a considerable time, even up to $30 \mathrm{sec}$ after the initial injection. Small angiomatous malformations show as well-defined localized areas of contrast pooling with a well-defined afferent arteriole.

\section{Malignant lesions}

The hepatoma produces a variety of arteriographic appearances and signs. At the one extreme there is the highly vascular solitary mass with marked enlargement of the hepatic and branch arteries, increased number, size and tortuosity of the arterioles and the production of small pools of contrast medium in the mass with irregular areas of increased density in the late phase. The adjacent vessels are displaced and compressed and these may also undergo considerable hypertrophy and tortuosity supplying the tumour as well. This is particularly so with the right phrenic artery in posterior tumours in the upper part of the right lobe of the liver. The extreme 


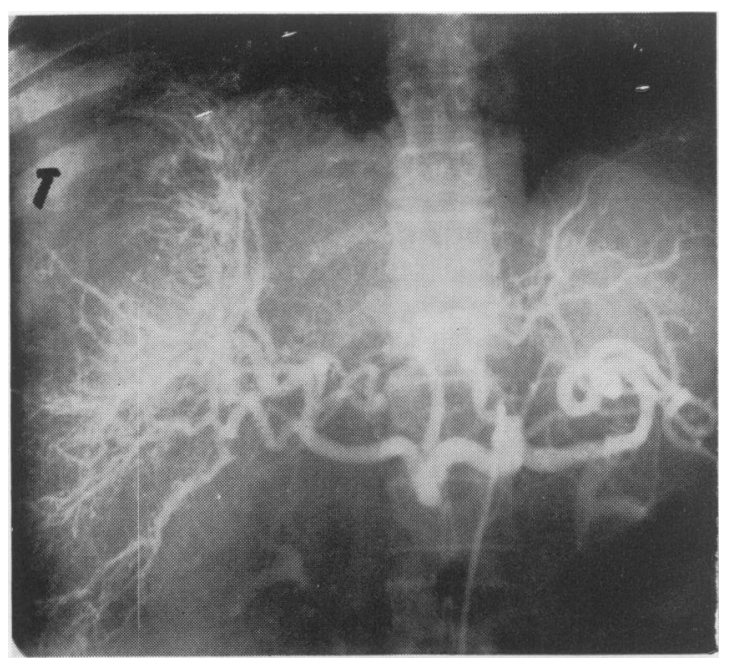

FIG. 8. Primary hepatoma (T) in a case of haemochromatosis occupying most of the right lobe of the liver with a central bare area, pathological radiating vessels at the margin and compression of adjacent arteries.

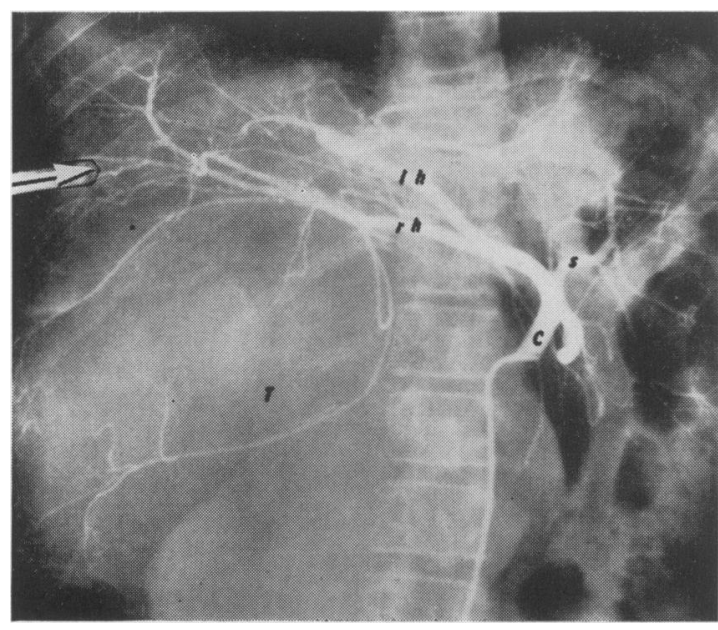

Fig. 9. Avascular hepatoma in the lower part of the right lobe of the liver displacing normal liver tissue upwards (arrow). T, Tumour; c, coeliac axis; s, splenic artery; rh, right hepatic artery; lh, left hepatic artery.

vascularity and contrast pooling may be present only in the more peripheral areas of the mass with relative avascularity of its more central part. The latter appearance is common with the hepatoma supervening in haemochromatosis (Fig. 8).

Another common appearance is that of a marked increase in vascularity due to re-duplication or triplication of the branch arteries and a marked increase in the smaller peripheral arteries of the liver.
This appearance is essentially due to obstruction of the intra-hepatic portal vein by the tumour. This pattern is easily recognizable when it affects a large area of the liver but can be extremely difficult to recognize when confined to the more peripheral part of the liver, especially at the top of the right lobe.

Occasionally the tumour may be shown as a filling defect in the portal vein, producing asymmetrical narrowing. With vascular tumours an actual arteriovenous shunt is produced and the portal vein shows densely in the arterial phase and can be seen to fill retrogradely. The filling defect or portal vein obstruction may, of course, also be shown by splenoportography.

The single primary malignant liver tumour can also be completely avascular (Fig. 9). This is especially so with the cholangioma. The ultrasonic scan is very helpful in this type of lesion, as it can distinguish solid from cystic masses. Arteriographically this type of lesion frequently produces the non-specific features of vascular displacement and a bare area. In some cases irregular infiltration of arterial walls or intra-hepatic arterial stenosis may be seen.

The hepatoblastoma can produce multiple, relatively discreet areas of hypervascularity with marked tumour staining in the late phase. This tumour maye be hormone secreting and has been associated with precocious puberty in children.

Hepatic metastases may be extremely large; solitary and vascular and in many respects resemble the solitary hepatoma (Fig. 10). The vascularity is, however, not usually as marked and the enlargement of the main hepatic artery is only slight. The smaller, or multiple, metastases frequently have a thick, dense margin with non-uptake of contrast in the centre of the lesion. The most vascular deposits arise from the colon, kidney, retro-peritoneal sarcoma and from the malignant melanoma. Carcinoid tumours and the insulinoma also produce vascular deposits, which may show as localized nodules of increased density arising from a feeding arteriole.

Infrequently, the liver lesions may be demonstrated prior to delineation of the primary tumour. If the examination is extended to include films after selective injection of the renal and superior mesenteric arteries, the original lesions may then be demonstrated (Fig. 11a and b).

\section{Vascular lesions}

Disease of the coeliac axis and mesenteric arteries is common but, fortunately, asymptomatic in the vast majority of cases. Unsuspected stenosis of the coeliac axis occurs in about $12 \%$ of cases and is a common cause of 'failure' of selective catheterization of this vessel. The usual cause is an atheromatous plaque near the orifice, but may be due to an extrinsic band, especially from the median arcuate 


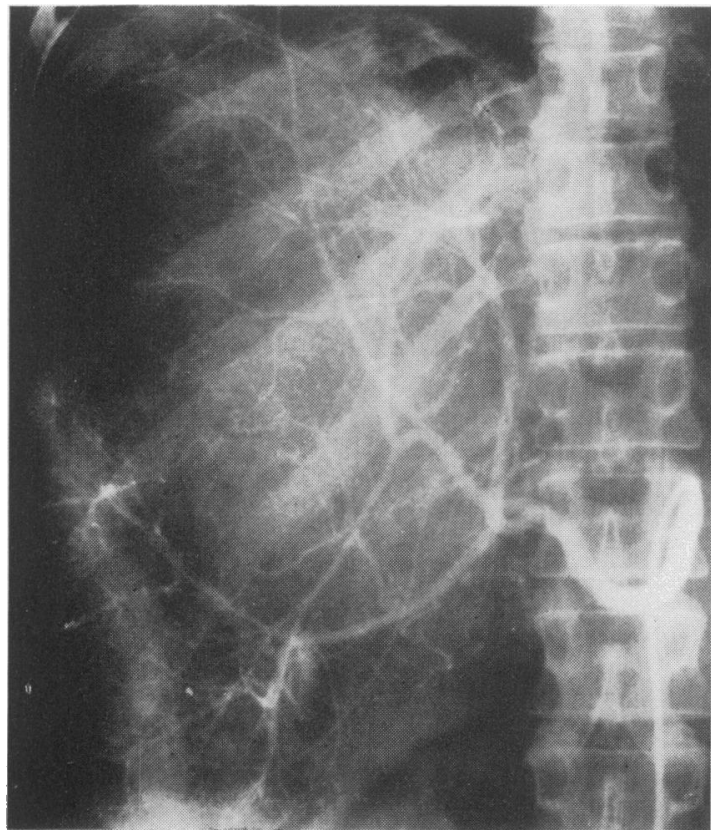

FIG. 10. Large single vascular metastasis from a colonic carcinoma producing marked displacement and spreading of hepatic arteries.

ligament of the diaphragm. The latter condition has been associated with abdominal 'angina' said to be caused by the 'steal' phenomenon. The blood supply to the superior mesenteric is diverted up the gastroduodenal to supply the hepatic and splenic vessels, producing relative anoxia of the intestine and causing post-prandial pain.

The appearance of this extrinsic lesion is characteristic in that the narrowing is a slight distance from the orifice and the coeliac axis is displaced towards the aorta at this point, producing an eccentric narrowing of the superior margin (Fig. 5).

Splenic artery aneurysms are the commonest incidental finding on coeliac axis arteriography. This lesion is particularly common in women over the age of 50 years, but fortunately rarely leads to symptoms, being well supported by peri-pancreatic and splenic hilar tissue. These aneurysms commonly calcify but very rarely rupture. Rupture or leak of the aneurysm may be a cause of upper abdominal pain, blood loss and shock. There is a particular predisposition for this to occur in the last trimester of pregnancy. A leaking splenic artery aneurysm has been diagnosed arteriographically. The particular interest in liver disease is that splenic artery aneurysms are extremely common in association with long-standing portal hypertension, irrespective of the cause (Fig. 12). Thus in the very large spleens of portal hypertension, intrasplenic bifurcation aneurysms will be found in $40 \%$ of cases on selective arteriography, but less commonly on the major artery. Again, these aneurysms seldom rupture.

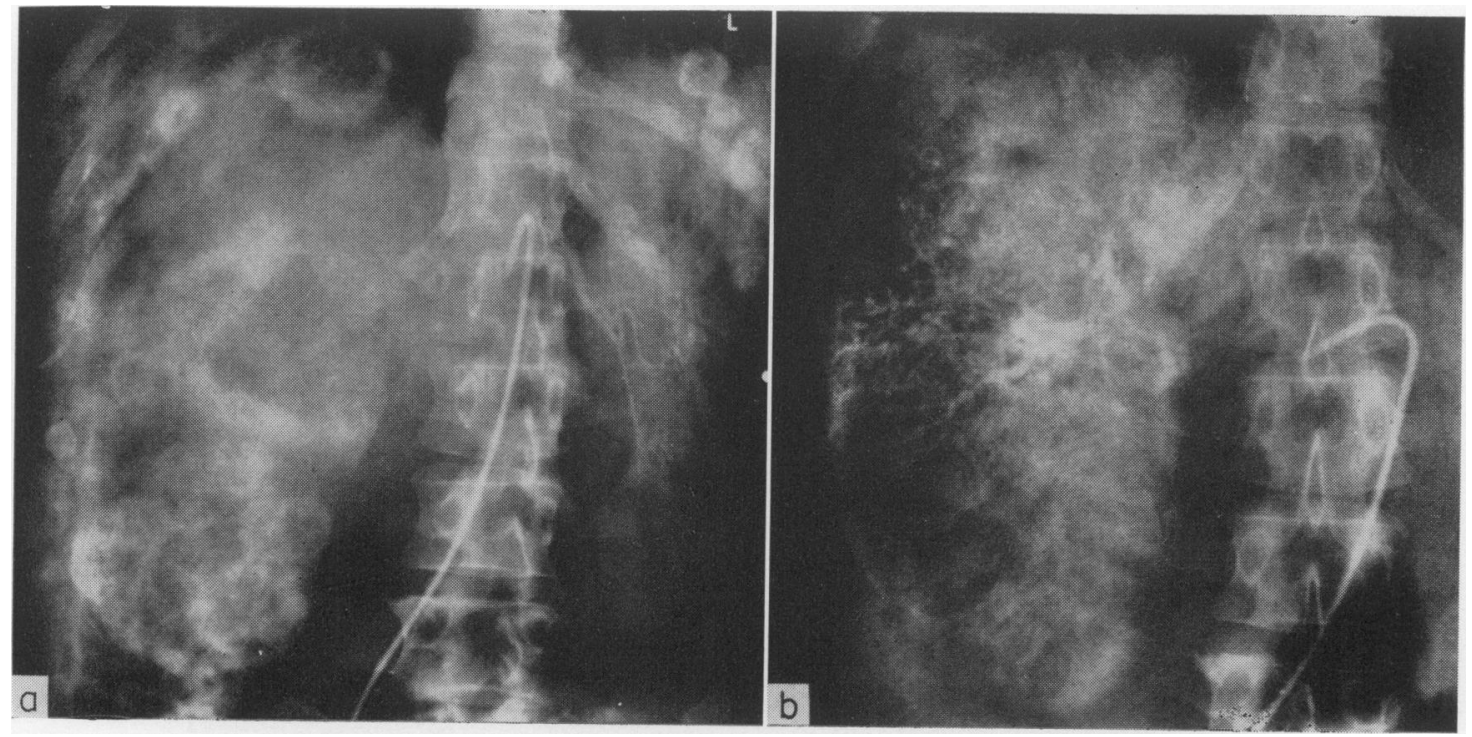

Fig. 11. (a) Multiple metastases in the liver in both the right and left lobes. The central areas are relatively avascular with a contrast blush at the periphery showing as a thick rim of tissue. (b) Late phase of selective right renal arteriogram showing a hypernephroma with appearances similar to the secondary deposits. 


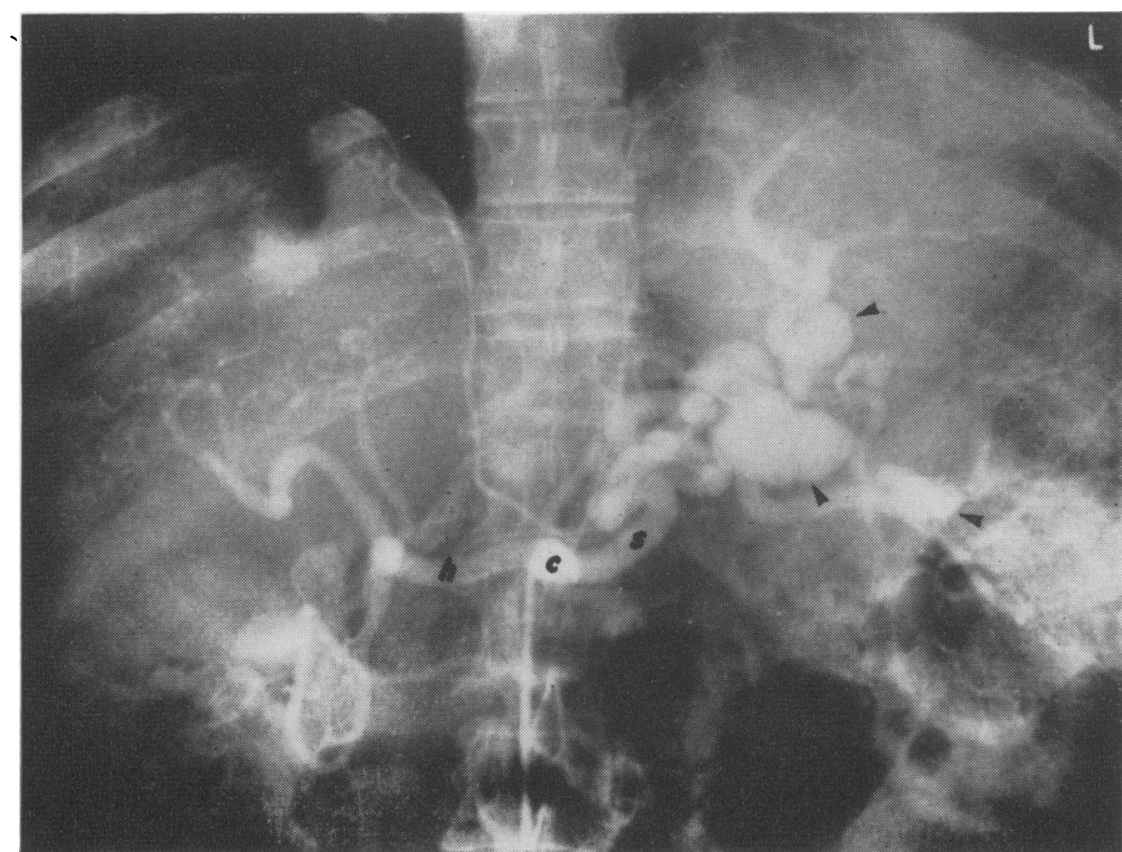

FIG. 12. There is a large aneurysm on the main splenic artery (s) and also small aneurysms on the branches at the hilum (h). Splenic artery aneurysms are commonly found in patients with portal hypertension (as in this case) but are usually intrasplenic and at bifurcations. c, Coeliac axis.

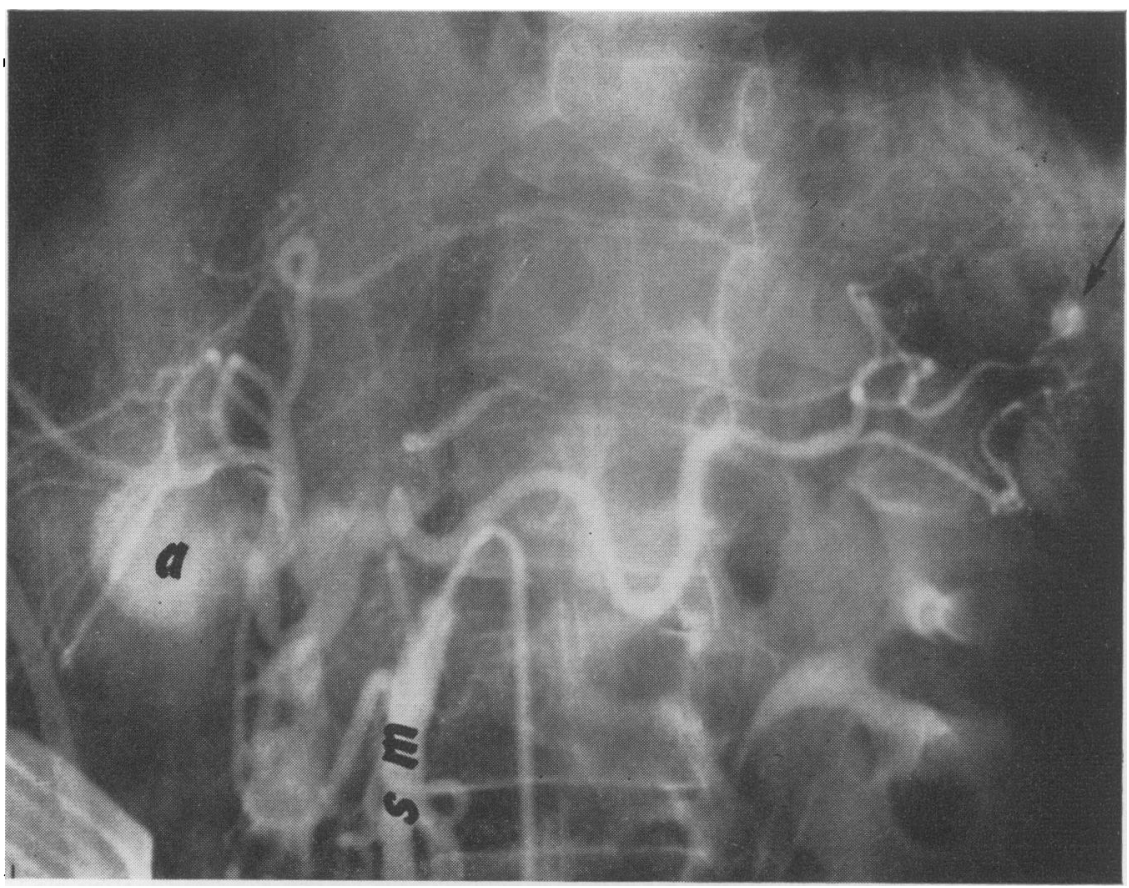

FIG. 13. Large aneurysm (a) of the hepatic artery which fills from the gastro-duodenal after injection into the superior mesenteric ( $\mathrm{sm}$ ) and then filling of the splenic artery with a small aneurysm (arrow). There was marked atheromatous stenosis of the coeliac axis. 
The main branch aneurysms may calcify, producing one or more curvilinear or egg-shell type calcifications in the left hypochondrium.

Hepatic artery (Fig. 13) and portal vein aneurysms also occur. These arteries are less well supported by surrounding adventitial tissue and have been associated with rupture. Aneurysms and stenosis may occur in association, particularly in the elderly.

A rare but important cause of portal hypertension is the arterio-venous fistula, most commonly occurring between splenic artery and vein. This lesion is well demonstrated on arteriography and when present offers the surgeon an opportunity of producing a complete cure of the portal hypertension.

\section{Arterio-venography in portal hypertension}

As was indicated previously, the pre-operative demonstration of the portal vein can be achieved by this method and the indications for using this procedure rather than spleno-portography have also been mentioned. The portal and mesenteric veins are never so densely shown as by spleno-portography and the collateral circulation is often difficult to see. However, these features can be enhanced by using a subtraction technique which increases visualization in the areas overlying the spine. As with splenoportography, a normal portal vein, a narrowed portal vein or cavernous transformation of the portal vein can be demonstrated.

In assessing the portal venous system as demonstrated on arterio-portography, it must be remembered that selective injection of the coeliac axis normally demonstrates the gastric and coronary veins, while demonstration of the superior mesenteric veins is usual by superior mesenteric arteriography. Unlike spleno-portography, the demonstration of these veins is thus not an indication of portal hypertension or a collateral circulation. For the radiographic diagnosis of a collateral circulation, veins must be shown which are not draining the area supplied by the injected artery, such as the inferior mesenteric vein in both coeliac and superior mesenteric arteriography or the mesenteric vein after selective injection of the splenic artery, indicating an abnormal direction of blood flow. Collateral vessels themselves are enlarged and often tortuous.

The arterial pattern is also visualized on arteriovenography and this shows definite changes associated with portal hypertension. There is a striking enlargement of the coeliac axis and splenic artery and the splenic artery often becomes extremely tortuous, but the hepatic artery does not enlarge unless some other lesion such as a hepatoma is present. As has been mentioned earlier, splenic artery aneurysms are also common under these circumstances and are probably the result of the prolonged increase in blood flow through the spleen.
In some of these cases there is a drop in the blood pressure in the coeliac axis even though no stenosis is present. This is probably due to the marked blood flow in this artery. By means of radioactive xenon studies done while a selective catheter was in the coeliac axis, it was possible to show that the increase in total blood flow through the spleen was of the order of three to five times greater than normal.

The hepatic arterial circulation appears to behave rather differently. In some cases of portal hypertension not only is there no concomitant enlargement of the hepatic artery as occurs in the coeliac and splenic, but there is a marked diminution in the contrast medium flow into the liver and down the gastro-duodenal artery, with poor visualization of
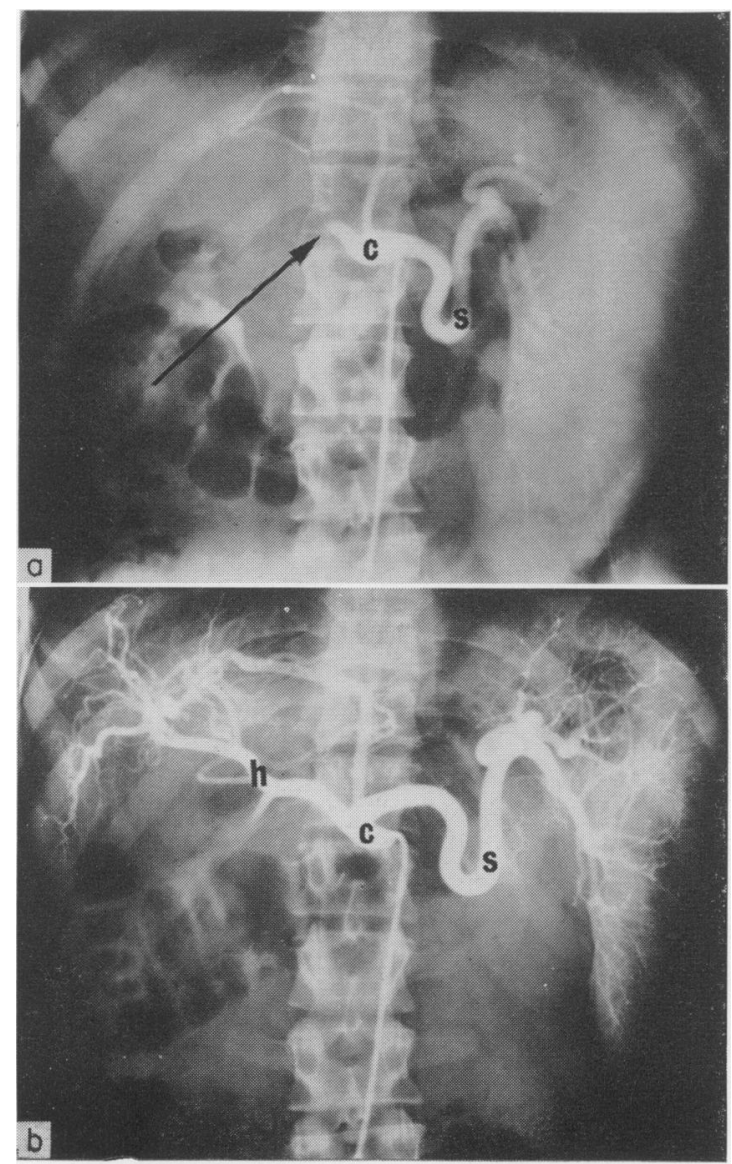

FIG. 14. (a) Coeliac axis (c) arteriography in portal hypertension prior to a portacaval shunt showing contrast medium up to the peripheral splenic (s) and phrenic vessels but only slight visualization of the hepatic artery (arrow). (b) Following a portacaval shunt there is prompt filling to the periphery of the liver as well as the spleen which has diminished in size. $h$, Hilum. 
the intrahepatic vessels (Fig. 14). In some of these cases it will be found that selective contrast injection into the superior mesenteric produces better filling of the hepatic artery than direct injection into the coeliac axis. There may even be filling of the splenic artery without a coeliac axis stenosis, indicating a reversed flow or possible 'steal' effect through the splenic circulation.

These changes in the hepatic arterial circulation can be shown to be functional by comparing the appearances before and after portacaval shunt. Following a successful portacaval shunt there is a markedly increased visualization of the hepatic circulation with a free and rapid flow of contrast medium to the peripheral hepatic vessels. Where xenon studies have been done after a successful portacaval shunt operation, it was found that there is no decrease in total blood flow through the spleen even though the spleen had decreased in size, indicating a fall in the intrasplenic peripheral resistance. This is in keeping with an earlier observation that the enlarged coeliac and splenic arteries of portal hypertension do not decrease in size following a successful shunt operation. It has also been noted that the diminished contrast visualization of the liver in portal hypertension does not occur in extrahepatic portal vein obstruction or in the presence of jaundice.

The most reasonable hypothesis at the moment to account for these phenomena appears to be that there is a veno-arterial reflex between the portal vein and the hepatic artery. Increased portal pressure acting on a normal venous wall initiates the reflex which causes constriction of the peripheral hepatic arteries. This reflex does not operate if the portal vein is thrombosed or thickened by periphlebitis and is obviated in the presence of jaundice. This reflex may also result in the diversion of blood flow to the splenic artery (rather than the splenic circulation being a cause of the 'steal' effect). Of course, if the constrictive hepatic arterial effects are irreversible, this would result in almost complete deprivation of liver blood supply if a portacaval shunt operation is carried out.

\section{Bibliography}

\section{Textbooks}

Maingot, R. (1969) Abdominal Operations, 5th edn. Appleton-Century-Crofts, New York.

Nebesar, R.A., Kornbith, P.L., Pollard, J.J. \& Michels, N.A. (1969) Celiac and Superior Mesenteric ArteriesA Correlation of Angiograms and Dissections. Churchill, London.

SCHOBINGER, R.A. \& RuzickA, F.F. (1964) Vascular Roentgenology. Macmillan, New York.

Shanks, S.C. \& Kerley, P. (1970) A Textbook of X-ray Diagnosis, 5th edn. H. K. Lewis, London.
SHERLOCK, S. (1968) Diseases of the Liver and Biliary System, 4th edn. Blackwell Scientitic Publications, Oxford and Edinburgh.

Smith, R. (1969) Progress in Clinical Surgery, Series III. Churchill, London.

Hepatic venography

Clain, D., Freston, J., Kreel, L. \& Sherlock, S. (1967) Clinical diagnosis of the Budd-Chiari syndrome. American Journal of Medicine, 43, 544.

Kreel, L., Freston, J.W. \& Clain, D. (1967) Vascular radiology in the Budd-Chiari syndrome. British Journal of Radiology, 40, 755.

Inferior vena-cavography

CONTO, D., JR \& Rocco, R.P. (1962) Inferior cavography in chronic fibrosing liver disease. Hospital, 61, 107.

Farinas, P.L. (1947) Abdominal venography. American Journal of Roentgenology, Radium Therapy, and Nuclear Medicine, 58, 599.

Fuchs, W.A. (1961) The diagnostic value of cavography. Radiologica clinica, 30, 129.

Umbilical vein and mesenteric portography

BAYLY, J.H. (1964) Use of umbilical vein in the diagnosis of upper gastro-intestinal bleeding. American Journal of Gastroenterology, 41, 235.

Bayly, J.H. \& Carbalhaes, O.G. (1964) Umbilical vein in adult: diagnosis, treatment and research. American Surgeon, 30, 56.

Carbalhaes, O.G. (1959) Hepatoportografia por via umbilical. Revista de sanidad militar, 12, 42.

Spleno-portography

LEGER, L. (1967) Spleno-portography. Thomas, Springfield, 응 Illinois.

Rousselot, L.M., Ruzicka, F.F., JR \& Doehner, G.A.ç (1956) Portography in portal hypertension; its application? in diagnosis and surgical planning. Surgical Clinics of North America, 36, 361.

Steiner, R.E., Sherlock, S. \& Turner, M.D. (1957) Percutaneous splenic portal venography. Journal of the Faculty of Radiologists, 8, 158.

Arteriography in liver disease (a) Technique

Evans, J.A. (1965) Techniques in the detection and diagnosis of malignant lesions of the liver, spleen and pancreas. Radiologic Clinics of North America, 3, 567.

KreEL, L. \& Williams, R. (1964) Arteriovenography of the portal system. British Medical Journal, 2, 1500.

Odman, P. (1956) Percutaneous selective angiography of the main branches of the aorta. Acta radiologica, 45, 1 .

Odman, P. (1958) Percutaneous selective angiography of the coeliac artery. Acta radiologica, Supplement 159.

Redman, H.C. \& Reuter, S.R. (1969) Angiographic demonstration of portacaval and other decompression liver shunts. Radiology, 92, 788.

Seldinger, S.I. (1953) Catheter replacement of needle in percutaneous arteriography: new technique. Acta radiologica, 39, 368.

(b) Space-occupying lesions

Abrams, R.M., Beranbaum, E.R., Santos, J.S. \& Lipson, J. (1969) Angiographic features of cavernous haemangioma of the liver. Radiology, 92, 308.

Baum, S., Roy, R., Finkelstein, A.K. \& Blakemore, W.S. (1965) Clinical application of selective coeliac and superior mesenteric arteriography. Radiology, 84, 279.

BERDON, W.E. \& BAKER, D.H. (1969) Giant haemangioma with cardiac failure in the newborn infant: value of high dosage intravenous urography and umbilical angiography. Radiology, 92, 1523. 
Evans, J.A. (1965) Techniques in the detection and diagnosis of malignant lesions of the liver, spleen and pancreas. Radiologic Clinics of North America, 3, 567.

FREDENS, M. (1969) Angiography in primary hepatic tumours in children. Acta radiologica, 8, 193.

Kreel, L. (1969) Radiology in liver disease. British Journal of Clinical Practice, 23, 187.

Kreel, L., Jones, E.A. \& Tavill, A. (1968) A comparative study of arteriography and scintillation scanning in spaceoccupying lesions of the liver. British Journal of Radiology, 41, 401.

Margulis, A.R., Nice, C.M. \& Rigler, L.G. (1956) Roentgen findings in primary hepatoma in infants and children: analysis of 11 cases. Radiology, 66, 809.

Milanes, B., McCook, J. \& Hernandez, A.L. (1953) Aortography and tumours of liver; preliminary report. Angiology, 4, 312.

Sammons, B.P., Neal, M.P., Armstrong. R.H., Jr \& Hager, H.G. (1967) Ten years experience with celicac and upper abdominal superior mesenteric arteriography. American Journal of Roentgenology, Radium Therapy, and Nuclear Medicine, 101, 345.

(c) Vascular and other lesions

Baum, S., Nusbaum, M., Clearfield, H.R., Kuroda, K. \& TUMEN, H.J. (1967) Angiography in the diagnosis of gastrointestinal bleeding. Archives of Internal Medicine, 119, 4. Boijsen, E., Judkins, M.P. \& SimaY, A. (1966) Angiographic diagnosis of hepatic rupture. Radiology, 86, 66.
Bron, K.M. \& Redman, H.C. (1969) Splanchnic artery stenosis and occlusion (incidence, arteriographic and clinical manifestations). Radiology, 92, 323.

Deutsch, V. (1968) Compression of the coeliac trunk and the angiographic evaluation of its haemodynamic significance. Clinical Radiology, 19, 309.

Harjola, P.T. \& LahtiharJu, A. (1968) Coeliac axis syndi ome. American Journal of Surgery, 115, 864.

(d) Portal hypertension

Boissen, F., Ekman, C.A. \& Olin, T. (1963) Coeliac and supe ior mesenteric angiography in portal hypertension. Acta chirurgica Scandinavica, 126, 315.

Gitlin, N., Grahame, G., Kreel, L. \& Sherlock, S. (1968) Arteriography, xenon blood flow and pressure studies in portal hypertension before and after portacaval shunt. Proceedings of the IIIrd Meeting European Association for the Study of the Liver, Modena, p. 22.

KREEL, L. (1967) The recognition and incidence of splenic artery aneurysms. M.D. Thesis, University of the Witwatersrand.

Kreel, L., Gitlin, N. \& Sherlock, S. (1969) Hepatic artery angiography in portal hypertension. American Journal of Medicine. (In press.)

RuzicKA, F.F. \& Rossi, P. (1969) Arterial portography: patterns of venous flow. Radiology, 92, 777.

Williams, R., Condon, R.E., Williams, H.S., Blendis, L.M. \& KreEL, L. (1968) Splenic blood flow in cirrhosis and portal hypertension. Clinical Science, 34, 441. 